\title{
Zur Kenntnis der Korksubstanz
}

\author{
(II. Mitteilung) \\ von \\ M. v. Schmidt.
}

Aus dem chemischen Laboratorium der $\mathrm{k} . \mathrm{k}$. Hochschule für Bodenkultur.

(Vorgelegt in der Sitzung am 17. Dezember 1903.)

Über den vermeintlichen Glyzeridcharakter der eigentlichen Korksubstanz.

Die in meiner ersten Abhandlung zitierte Arbeit Kügler's gibt der Vermutung Raum, daß sowohl der Chloroformextrakt, als auch der nur durch alkoholisches Kali ausziehbare Anteil des Korkes im wesentlichen aus den Glyzerinestern der Phellonsäure und anderer Fettsäuren besteht.

Das Vorhandensein von Fettsäuren hier wie dort konnte ich bestätigen; unwahrscheinlich aber schien mir die Annahme, daß diese in beiden Fraktionen als identische Verbindungen auftreten. Wäre dies der Fall, so könnte ich mir nicht erklären, weshalb der Kork auch im Zustande unfühlbarer Verteilung nur etwa $10 \%$ derselben an indifferente Lösungsmittel abgibt, während alkoholisches Kali den über $30 \%$ betragenden Rest mit Leichtigkeit völlig extrahiert.

Küg1er führt dieses Verhalten darauf zurück, daß die Moleküle des Korkfettes zwischen den Zellulosemolekülen eingebettet liegen und daß diese letzteren dem Eindringen des Lösungsmittels einen energischen Widerstand entgegensetzen; alkoholisches Kali hingegen durchdringe die Zellulose, indem es in geringem Grade verändernd darauf einwirkt. 
Diese Erklärung erscheint mir schon durch die von Kügler selbst mitgeteilte Tatsache widerlegt, daß eine $2 \mathrm{~cm}$ dicke Korkscheibe nach mehrtägiger Mazeration in Chloroform durchscheinend wird, denn dies ist offenbar nur möglich, wenn eine vollständige Durchtränkung mit dem Lösungsmittel stattfindet. Außerdem steht sie in Widerspruch mit den Untersuchungen v. Höhnel's, nach welchen sich die leicht extrahierbaren Cerinkrystalle in der Zelle seibst und als dünner Überzug der inneren Lamelle - des Zelluloseschlauches - vorfinden. Wenn also das Lösungsmittel bis in das Lumen der Zelle gelangt, so muB es zuvor die Holz- und Suberinlamelle durchdringen und unter diesen Umständen müßten auch die dort abgelagerten Substanzen völlig entfernt werden können, soferne sie überhaupt löslich sind.

Das auffällige Verhalten des Korkes gegen Lösungsmittel erklärt sich aber ganz ungezwungen, wenn man annimmt, daß die Korkfettsäuren in zwei verschiedenen Verbindungsformen zugegen sind: Ein kleiner Teil derselben in Gestalt von leicht löslichen Glyzeriden, die Hauptmenge aber in anderer Form - vielleicht als verseifbare Anhydride -, die den meisten Lösungsmitteln widerstehen und daher als solche nicht isoliert werden können.

Um völlige Klarheit in diese Verhältnisse zu bringen, waren folgende Fragen zu beantworten:

1. Enthält der Kork überhaupt Glyzeride?

2. Ist auch in dem mit Lösungsmitteln erschöpften Kork (der eigentlichen Korksubstanz) gebundenes Glyzerin enthalten?

3. Steht die Menge des Glyzerins in einem angemessenen Verhältnisse zur Quantität der Fettsäuren?

Von diesen Fragen hat Kügler nur die erste und auch diese nicht in ganz einwandfreier Weise beantwortet. Er erhielt aus den nach der Verseifung des Korkes und Abscheidung der Fettsäuren resultierenden Laugen durch Eindampfen und Extraktion des Salzrückstandes mit Alkohol einen gelb gefärbten Sirup, den er durch folgende Reaktionen mit Glyzerin identifizieren zu können glaubte.

1. Ein Tropfen dieser Flüssigkeit, mit einem Stückchen Kaliumbydrosulfat erhitzt, entwickelte Acroleïndämpfe. 
2. Beim Erhitzen mit Oxalsäure auf $100^{\circ}$ entwich Kohlendioxyd; auch konnte im Destillate des mit Wasser verdunnten Rückstandes Ameisensäure nachgewiesen werden.

3. Durch Behandlung mit Salpeterschwefelsäure entstand ein in Wasser unlösliches Öl, das explosive Eigenschaften zeigte.

4. Eine mit der alkalisch gemachten Flüssigkeit befeuchtete Boraxperle färbte die Flamme grün.

Von den hier angegebenen Reaktionen kann wohl nur die Acroleïnprobe als beweiskräftig gelten, obgleich auch bei dieser Täuschungen nicht ganz ausgeschlossen sind. Der Zerfall der Oxalsäure in Kohlendioxyd und Ameisensäure kann durch gar viele organische Substanzen und auch durch bloßes Erhitzen ohne diese veranlaßst werden und die Entstehung eines explosiven Körpers ist für die Gegenwart von Glyzerin nicht gerade charakteristisch. Auch die Grünfärbung der Flamme durch die Boraxperle flößt kein besonderes Vertrauen ein, da bekanntlich Erythrit und Mannit dasselbe Verhalten zeigen. Endlich kann der Befund Kügler's ebenso gut auf mangelhafte Entfernung extrahierbarer Glyzeride aus der eigentlichen Korksubstanz zurückgeführt werden.

Angesichts dieser Sachlage hielt ich es für geboten, den Nachweis des Glyzerins neuerdings zu versuchen und durch eine Quantitätsbestimmung zu ergänzen.

$\mathrm{Zu}$ diesem $\mathrm{Zwecke}$ wurde feinst geraspelter Kork durch feine Müllergaze gebeutelt und der erhaltene Staub der Reihe nach mit Chloroform, Alkohol, Sodalösung und Wasser erschöpfend extrahiert. Was hiebei ungelöst blieb, wurde mit alkoholischer Kalilauge verseift, der Alkohol abdestilliert, die Seife in Wasser aufgenommen und mit Essigsäure zersetzt. Das Gewicht der hieaurch abgeschiedenen Fettsäuren betrug $1.6 g$.

Zur Berechnung des an diese Fettsäuren möglicherweise gebunden gewesenen Glyzerins wurden dieselben in Alkohol gelöst und unter Zusatz von Phenolphtaleïn mit Kalilauge titriert. Zur Neutralisation waren $13.7 \mathrm{~cm}^{3}$ Lauge $\left(1 \mathrm{~cm}^{3}=\right.$ $0.02369 \mathrm{~g} \mathrm{KOH}$ ), enthaltend $0.32455 \mathrm{~g}$ Kalihydrat erforderich. Unter der Voraussetzung, daß je 3 Moleküle Ätzkali einem 
Molekül Glyzerin entsprechen, berechnet sich dessen Menge mit $0 \cdot 1779 g$.

Die von den Fettsäuren getrennte saure Flüssigkeit wurde zur Bestimmung des Glyzerins nach $Z$ eisel und $F$ an to ${ }^{1}$ verwendet. Beim Einkochen dieser Lösung im schief gestellten Glaskolben schieden sich gelbe Flocken ab, die durch Filtration entfernt wurden. Bei weiterer Konzentration fielen neue Mengen desselben Körpers aus und nach dessen Entfernung ein drittes Ma1. Schließlich erhielt ich eine klare, aber deutlich braungelb gefärbte Flüssigkeit, deren Volum auf $50 \mathrm{~cm}^{3}$ gebracht wurde.

$5 \mathrm{~cm}^{3}$ derselben, in den Glyzerinbestimmungsapparat gebracht, gaben $0 \cdot 064 g$ Jodsilber, entsprechend $0 \cdot 0251 g$ Glyzerin. Rechnet man dieses auf die Gesamtlösung um, so ergibt sich der Glyzeringehalt derselben mit $0.251 \mathrm{~g}$.

Vergleicht man diese Menge mit der aus dem Kaliverbrauch berechneten $(0 \cdot 177)$, so ergibt sich, daß die Lösung - wenn überhaupt Glyzerin darin enthalten war - noch andere Körper enthaiten mußte, die unter diesen Umständen gleichfalls Jodsilber liefern. Tatsächlich erhielt ich aus dem vorerwähnten schwer löslichen Körper, der sich beim Konzentrieren der Glyzerinlösung abschied, neue Mengen von Jodsilber.

Um ihn vollständig zu entfernen und die Glyzerinbestimmung korrekter zu gestalten, wurde der Rest der Flüssigkeit mit frisch bereitetem Bleiessig gefällt, das Filtrat mit Natriumphosphat entbleit und abermals auf $50 \mathrm{~cm}^{3}$ eingelxocht. Es war jetzt erheblich heller, aber immer noch gelblich gefärbt. Aus $5 \mathrm{~cm}^{3}$ desselben ( $=9 / 100$ der ursprünglichen Menge) bekam ich $0.0398 \mathrm{~g}$ Jodsilber, entsprechend $0.01561 \mathrm{~g}$ Glyzerin, woraus sich der Glyzeringehalt der ursprünglichen Gesamtlösung mit $0 \cdot 1405 \mathrm{~g}$ berechnet.

Wie ersichtlich stimmt dieser mit dem aus der Säurezahl der Fettsäuren berechneten $(0 \cdot 1779 \mathrm{~g})$ annähernd überein, denn der Fehlbetrag ist durch die vielfachen Operationen, welche die Flüssigkeit durchzumachen hatte, leicht erklärt.

1 Zeitschrift für das landwirtsehaftliche Versuchswesen in Österreich, 1902 . 
Es hatte somit den Anschein, als ob tatsächlich die Fettsäuren des Korkes - wenigstens in dem mit Lösungsmittein erschöpften Teile desselben - in Form von Glyzerinestern vorhanden wären. Einwandfrei jedoch war die Bestimmung keineswegs, da die Jodsilber liefernde Substanz nicht notwendigerweise Glyzerin gewesen sein mußte, sondern vielleicht ein anderer, durch Bleiessig nicht vollständig entfernbarer Körper war.

Um diese Bedenken zu zerstreuen, versuchte ich, den Rest der vermeintlichen Glyzerinlösung nach dem Vorgang von Törring ${ }^{1} \mathrm{zu}$ destillieren. Zur Vermeidung des Überspritzens der stark schäumenden Flüssigkeit wurde die Destillation zunächst bei Wasserbadtemperatur unter sehr mäßiger Druckverminderung vorgenommen, dann erst die Retorte in ein Luftbad gesenkt und die Operation bei vollem Vakuum der Wasserstrahlpumpe und einer Temperatur von $220^{\circ}$ vollendet. Der trockene Retorteninhalt wurde mit etwa $10 \mathrm{~cm}^{3}$ Wasser übergossen und dieses ein zweites $\mathrm{Mal}$ in gleicher Weise abdestilliert.

Das mit den Spülwässern vereinigte Destillat war farblos. Auf $50 \mathrm{~cm}^{3}$ eingeengt, zeigte es einen deutlich gelben Stich. $5 \mathrm{~cm}^{3}$ desselben wurden wie zuvor in den Apparat gebracht und mit Jodwasserstoffsäure gekocht. Das Ergebnis war überraschend. Ich erhielt wohl eine Opaleszenz der Silberlösung, die aber so unbedeutend war, daß durch den bloßen Augenschein auf die Abwesenheit des Glyzerins geschlossen werden konnte. Eine Wägung der abgeschiedenen Trübung bestätigte diese Vermutung, denn sie ergab das Vorhandensein von nur $2 \frac{1}{2} m g$ Jodsilber. Nimmt man an, daß dieses aus Glyzerin entstanden war, so könnten in der zum Versuche verwendeten Lösung nur $0.98 \mathrm{mg}$ enthalten sein, also in der ursprünglichen Gesamtflüssigkeit $12 \cdot 4 \mathrm{mg}$, die zu der entsprechenden Menge Fettsäuren $(1.6 \mathrm{~g})$ in gar keinem Verhältnisse stehen.

Zum Ubberflusse wurde die Abwesenheit des Glyzerins auch durch die Acroleinprobe festgestellt. Der Rest der Lösung wurde bis auf etwa $1 \mathrm{~cm}^{3}$ eingekocht, dann im Proberöhrchen

1 Landwirtschaftl. Versuchsstationen, $36,29$. 
unter Zusatz von Kaliumhydrosulfat zur Trockene gebracht und schließlich die Salzmasse bis zum Schmelzen erhitzt. Wie vorauszusehen war, konnte hiebei nicht der geringste Acroleïngeruch wahrgenommen werden. Da nach Versuchen von $Z$ eisel und Fanto ${ }^{1}$ auf diesem Wege noch die Gegenwart von $4 \mathrm{mg}$ Glyzerin überzeugend nachgewiesen werden kann, so war ich wohl berechtigt, meine Lösung als glyzerinfrei $\mathrm{zu}$ betrachten.

Man könnte einwenden, daß die Anwesenheit größerer Mengen von Salzen und organischen Beimengungen das Übergehen des Glyzerins erschwerte und etwaige fehlerhafte Anordnung des Destillierapparates das ihrige dazu beitrug, die Ausbeute zu vermindern. Es war sonach die Abwesenheit des Glyzerins erst dann überzeugend konstatiert, wenn der Nachweis gelang, daß Glyzerin unter denselben Umständen doch der Hauptmenge nach überdestilliert.

Die aus Kork gewonnene mutmaßliche Glyzerinlösung enthielt relativ große Mengen von Natriumacetat und unbekannte, durch Bleiessig fällbare organische Substanzen. Um daher die Versuchsbedingungen möglichst ähnlich zu gestalten, wurde die Retorte mit einer reinen Glyzerinlösung von bekanntem Gehalte beschickt, deren Menge so bemessen wurde, daß darin $0.1405 \mathrm{~g}$ Glyzerin (also ebenso viel, als sich anscheinend aus der Analyse der mit Bleiessig gereinigten, aber nicht destillierten Lösung ergeben hatte) enthalten war. Dazu wurde $1 g$ Natriumacetat und ebenso viel Tannin ${ }^{2}$ gebracht, was ungefähr der Menge des zuvor erhaltenen Destillationsrückstandes entsprach. Die Destillation wurcle in derselben Retorte und demselben Luftbade bei gleicher Temperatur vorgenommen. Auch wurde die Zeitdauer und Luftverdünnung eingehalten, die beim ersten Versuch zur Anwendung kam. Das so erhaltene Destillat wurde wieder auf $50 \mathrm{~cm}^{3}$ gebracht und auf seinen Glyzeringehalt geprüft. Ich erhielt aus $5 \mathrm{~cm}^{3}$

I Miindliche Mitteilung.

2 Da mir über die in den Verseifungslaugen des Korkes enthaltenen organischen Substanzen nichts weiter bekannt war, als daB sie durch Bleiessig fällbar sind, so wählte ich auf gut Glück Tannin zu ihrer Substitution, das sich wenigstens in dieser Hinsicht ebenso verhält. 
desselben $0.035 \mathrm{~g}$ Jodsilber, entsprechend $0.01373 \mathrm{~g}$ Glyzerin. Auf die Gesamtflüssigkeit umgerechnet, ergibt sich der Glyzeringehalt des Destillates mit $0.1373 \mathrm{~g}$ gegen $0.1405 \mathrm{~g}$ der zum Versuch verwendeten Substanz.

Da sonach unter denselben Umständen fast alles Glyzerin überging, so kann aus dem negativen Ergebnisse des vorerwähnten Versuches wohl mit Sicherheit geschlossen werden, daß die eigentliche Korksubstanz nahezu frei davon ist.

Um die Frage zu entscheiden, ob der durch Chloroform ausziehbare Teil des Korkes Glyzerin enthält oder nicht, wurde zunächst eine kleine Menge dieses Extraktes mit Wasser anhaltend gekocht, um eine möglicherweise gebildete Chloroformverbindung zu zersetzen, sodann getrocknet und ohne weitere Vorbereitung in den Apparat gebracht.

$0.1604 \mathrm{~g}$ dieser Substanz gaben $13 \mathrm{mg}$ Jodsilber. Nimmt man an, daß die Jodsilber liefernde Substanz Glyzerin war, so müßte die abgewogene Menge $5 \mathrm{mg}$ davon enthalten. Diese geringe Menge hat nichts Auffälliges, wenn man erwägt, daß gut ein Dritteil des Chloroformextraktes aus Cerin besteht, das - wie ich mich überzeugte - absolut kein Jodsilber gibt, und daß neben diesem auch noch andere Körper vorhanden sind, die sich in dieser Hinsicht indifferent verhalten. Überdies werden nach Zeisel und Fanto ${ }^{1}$ Glyzeride durch Jodwasserstoffsäure nicht vollständig zersetzt, so daß die Ausbeuten an Glyzerin nur 75 bis $80 \%$ betragen. Jedenfalls hatte dieser Vorversuch die Möglichkeit der Gegenwart von Glyzerin ergeben, so daß dessen exakte Bestimmung versucht werden mußte.

Sonach wurden $1 \cdot 445 \mathrm{~g}$ des Chloroformextraktes mit alkoholischem Kali verseift, der Alkohol abdestilliert und der Rückstand mit Essigsäure zerlegt. Die von den Fettsäuren getrennte Flüssigkeit wurde auf ein kleines Volum eingekocht, hierauf in der oben beschriebenen. Weise destilliert. Das Destillat wurde auf $50 \mathrm{~cm}^{3}$ gebracht. $5 \mathrm{~cm}^{3}$ desselben gaben $0.0036 \mathrm{~g}$ Jodsilber. Es hätte sonach die Gesamtflüssigkeit $36 \mathrm{mg}$ ergeben, was einem Glyzeringehalt von $14 \mathrm{mg}$ entspricht. 
Nimmt man den durchschnittlichen Glyzeringehalt der Fette mit $10 \%$ an, so wären in der genannten Extraktmenge $0.14 \mathrm{~g}$ Glyzeride, d. i. ziemlich genau 10\% der Substanz.

Da bei Anwendung so geringer Substanzmengen die unvermeidlichen Versuchsfehler schon stark ins Gewicht fallen, so versuchte ich die Gegenwart des Glyzerins mit Zuhilfenahme größerer Quantitäten auf indirektem Wege festzusteilen. Waren nämlich die im Chloroformauszug enthaltenen Fettsäuren an Glyzerin gebunden, so mußte ihr Gewicht durch die Verseifung um $19 / 46$ des vorhandenen Glyzerins vermindert werden. Sind hingegen nur freie Fettsäuren oder wachsartige Verbindungen zugegen, so darf ihr Gewicht hiedurch entweder gar keine Veränderung erleiden oder muß um ein Geringes zunehmen.

Zur Vornahme dieses Versuches wurden $3.6575 \mathrm{~g}$ des über Phosphorpentoxyd getrockneten Extraktes wie zuvor verseift, das entstandene Produkt mit verdünnter Schwefelsüure zerlegt und die so erhaltenen Fettsäuren durch ein pergamentisiertes Filter von der Flüssigkeit getrennt. Der Filterinhalt wurde mit Wasser erschöpfend gewaschen. Nachdem er lufttrocken geworden war, konnte er ohne Verlust vom Papier entfernt und in ein Wägefäschchen gebracht werden, worauf er, um eine mögliche Oxydation zu verhindern, gleichfalls im Vakuum über Phosphorpentoxyd getrocknet wurde. Ich erhielt auf diese Weise $3.319 g$ Fettsäuren. Der Gewichtsverlust betrug sonach $0.3385 \mathrm{~g}$, d. i. etwas über $10 \%$.

Daß dieser nur zum kleinsten Teile auf Rechnung des Glyzerins zu setzen ist, bewiesen neben dem Ergebnisse der oben angeführten direkten Bestimmung auch die stark braun gefärbten Mutterlaugen, welche auf die gleichzeitige Anwesenheit von organischen Substanzen hinwiesen, die weder Glyzeride noch Wachsarten sein können. Der Versuch war also aus diesem Grunde nicht konkludent, doch steht sein Ergebnis zum mindesten nicht in Widerspruch mit der vermuteten Gegenwart des Glyzerins. Dagegen gelang dessen Nachweis durch die Acroleïnprobe.

Zur Vornahme dieser wurde die von den Fettsäuren getrennte saure Flüssigkeit auf ein kleines Volum eingekocht und in der zuvor geschilderten Weise mit Kaliumhydrosulfat 
erhitzt. Hiebei entstanden Acroleindämpfe in solcher Menge, daß an dem Vorhandensein des Glyzerins nicht mehr gezweifelt werden konnte.

Zusammenfassung: 1. Der durch Chloroform extrahierbare Teil des Korkes enthält neben Cerin und anderen nicht näher untersuchten Körpern auch Glyzeride von Fettsäuren in nicht zu vernachlässigender Menge. 2. Die eigentliche Korksubstanz ist frei von Glyzeriden oder enthält nur so geringe Mengen derselben, daß diese zu den hier in anderer Bindungsform vorhandenen Fettsäuren auch nicht entfernt in einem komensurablen Verhältnisse stehen.

In welcher Form die Hauptmenge der Fettsäuren im Suberin enthalten ist, kann wegen der Unmöglichkeit, sie in ihrem nativen Zustande zu isolieren, auf direktem Wege wohl kaum festgestellt werden. Doch läßt sich vermuten, daß man es hier mit verseifbaren Anhydriden zu tun hat. $\mathrm{Ob}$ diese bereits im frischen Kork präformiert enthalten oder erst posthum entstanden sind, ließe sich durch Untersuchung des Korkes in verschiedenen Altersstadien vielleicht erweisen. Wenn man erwägt, daß Flaschenkork 7 bis 15 Jahre alt werden muß, bevor er geerntet werden kann, so ist wohl die Möglichkeit gegeben, daß die ursprünglich darin enthaltenen Substanzen bis dahin ihre Zusammensetzung ändern. 